\title{
Identifying Patient-Ventilator Asynchrony Using Waveform Analysis
}

\author{
Ivan I Ramirez ${ }^{1}$, Daniel H Arellano ${ }^{2 *}$ \\ ${ }^{1}$ PT, Division of Critical Care Medicine, Hospital Clinico Universidad de Chile, Santiago. Universidad San Sebastian, Santiago, \\ Chile and Universidad de los Andes, Santiago, Chile \\ ${ }^{2}$ PT CRT MSc, Division of Critical Care Medicine, Hospital Clinico Universidad de Chile and Respiratory Care Laboratory, \\ Kinesiology Department, Santiago of Chile and Universidad San Sebastian.
}

Received: May 05, 2017; Accepted: June 07, 2017; Published: December 04, 2017

*Corresponding author: Daniel H Arellano PT CRT MSC, Intensive Care Unit, Hospital Clinico Universidad de Chile, Santos Dumont 999, Independencia, Santiago,Chile.Email: darellano@vtr.net ; daskineint@gmail.com

\begin{abstract}
A significant percentage of mechanically ventilated patients in Intensive Care Units (ICUs) show some type of patient-ventilator asynchrony (PVA). The presence of PVA is associated with complications that affect the clinical outcome and the goals for which mechanical ventilation is used in critically ill patients. Currently, mechanical ventilators are able to show different types of waveforms that allow to identify the different types of PVA in a noninvasive and reliable way. However, in order to perform an adequate interpretation and management of the PVA the health care professionals must be properly trained in the topic.
\end{abstract}

\section{Introduction}

Mechanical ventilation is used in intensive care units (ICUs) in order to decrease the work of breathing, maintain the adequate gas exchange, and unloading the respiratory muscles [1-3]. However, these goals can be difficult to achieve if there is not an adequate interaction between patient and ventilator, which is known as patient-ventilator asynchrony (PVA)3. Epstein2 defines PVA as "any condition where patient-ventilator interaction is not optimal."

Based on evidence, it is necessary to consider that a significant percentage of patients will present some type of asynchrony during mechanical ventilation (approximately 25\%) [4]. For that reason, health care professionals should be aware, especially when sedation levels are lowered, active humidification systems are used and when parameters are selected, in order to ensure an appropriate interaction and to prevent complications associated with PVA [Table 1].

Currently, mechanical ventilators used in ICUs show different types of waveforms, such as: pressure/time, flow/time and volume/time. One of the main reason why current ventilators show this visual information is to identify if there is an adequate interaction between the patient and the ventilator5. Identifying PVA using waveform analysis, is a non invasive and reliable method, which has shown to have a good correlation with other methods such as the identification of PVA by measurement of the esophageal pressure [4].

Several authors point out that the interpretation of PVA using waveform analysis, is a skill that every health care professional who is in contact with ventilated patients, should develop in order to prevent their appearance and their complications associated [3,5-9] [Table 1]. However, the interpretation of PVA is a skill that requires specific training. For example, in some cases health care professionals may identify more than one type of PVA in the ventilator graphics at the same time, which make it more difficult to analyze. Therefore, the identification of PVA is not an easy task. Ramírezet al [10] evaluated the ability of 366 professionals that work in ICUs to identify PVA using waveform analysis. Their results showed that only $21 \%$ of health care professionals were able to recognize all types PVAs. In addition, they demonstrated that neither experience nor profession proved to be a relevant
Table: 1
Complications associated to Patient-Ventilator Asynchrony (PVA)
- Increased work of breathing.
- Ineffective effort.
- $\quad$ Air trapping.
- Respiratory alkalosis and hyper inflation of the lungs (Auto-
triggering).
- Dynamic Hyper inflation.
- $\quad$ Auto PEEP (also known as intrinsic PEEP (PEEPi)).
Alters the outcome of Weaning.
- Increased levels of sedation.
- Confusion about the actual condition of the patient.
- $\quad$ Sleep disorders.
- $\quad$ Longer stay in Intensive Care Units and mechanical ventilation. 
factor to identify asynchrony correctly using waveform analysis. However, they found that health care professionals who have specific training in mechanical ventilation increase their ability to identify asynchrony using waveform analysis.

For this reason, the objective of this article is to review the types of PVAs, their effects on the mechanically ventilated patient and their analysis based on the mechanical ventilator waveforms.

\section{Asynchrony Index}

The Asynchrony Index (AI) is defined as the number of asynchrony events divided by the total respiratory rate (Number of asynchronies / Number of total respiratory rate (Number of asynchrony events + Number of cycles effectively delivered by the mechanical ventilator) x 100 [11].

The AI has been used in different studies to evaluate the effect and complications associated with PVAs. The value is expressed as a percentage and values $\geq 10 \%$ are associated with complications $[4,12,13]$. The complications associated to PVA are summarized in Table 1.

\section{Asynchronies Related To Trigger}

Asynchronies related to trigger (the variable that start[s] inspiration) are the most frequent and studied type of PVAs in ventilated patients [4]. Chao et al compared a group of patients who presented PVA related to trigger vs. A group that did not have this type of PVA [12]. The results showed that only $16 \%$ of the patients in the group with PVA related to trigger, had a successful weaning process versus a $57 \%$ of success in the group of patients who did not have PVA. It was also observed that in the first group the average duration of the weaning process (in days) was significantly higher compared to the second group, which can be translated into a greater number of days in mechanical ventilation and ICU.

Within the group of asynchronies related to trigger are: ineffective efforts, auto-triggering, double triggering and reverse trigger.

\section{Ineffective Efforts}

Ineffective effort is defined as "patient efforts that are not sensed by the ventilator" [6]. In other words, the patient generates an inspiratory effort, but the ventilator does not recognize it and does not deliver a breath to the patient. The main characteristic of ineffective efforts is to produce an airway pressure drop in the pressure/time waveform, caused by the inspiratory effort of the patient, which decreases the airway pressure and, a change in the expiratory flow (which tends to return to zero due to the inspiratory effort of the patient) without the delivery of a breath from the ventilator [4,14][Figure 1]. This type of PVA is the most frequent and occurs more frequently during the expiratory phase (may also occur during the inspiratory phase), in all modes $[4,15]$.

Thilleet al found that Ineffective triggering and doubletriggering accounted for more than $98 \%$ of the total number of asynchrony events (85\% were ineffective triggering events, and $13 \%$ were double-triggering events)[4]. Among ineffective triggering events, $78 \%$ occurred during the expiratory period

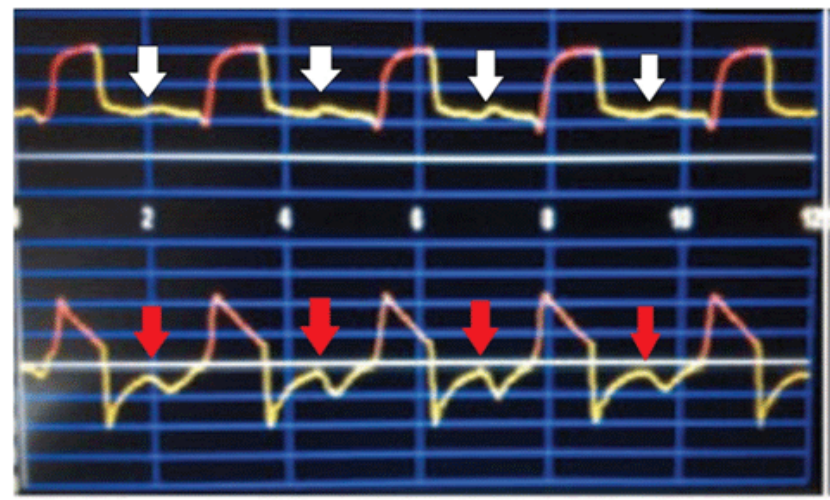

Figure 1: White arrows show ineffective efforts in the pressure/time waveform. Red arrows show ineffective efforts in the flow/time waveform

and $7 \%$ during the inspiratory phase. De Wit et al obtained similar results, where ineffective efforts accounted for $88.3 \%$ of total PVAs [16].

This type of PVA is common in patients with chronic obstructive pulmonary disease (COPD), as they are patients who must generate a great inspiratory effort to overcome the Auto-PEEP, also known as intrinsic positive end-expiratory pressure (PEEPi) $[4,9]$. On the other hand, these patients have a short expiratory time which may cause air trapping, dynamic hyperinflation and Auto-PEEP.

However, there are other causes of ineffective efforts; for example, Inadequate programming of triggering sensitivity and the level of pressure support $[4,12,17]$. Leung et al demonstrated that as the level of pressure support increases the respiratory drive decreases, resulting in breaths with a higher tidal volume, longer inspiratory time and shorter expiratory time, which are the characteristics of the breaths that precede ineffective efforts, leading to air trapping, dynamic hyperinflation, Auto-PEEP and ineffective efforts [17].

\section{Auto-Triggering}

Auto-triggering is a type of PVA that can be caused by leaks in the mechanical ventilator circuit, condensation in the circuit, improper setting of sensitivity and cardiac oscillations $[6,14,19]$. Auto-triggering is defined as "a delivery of a breath that is neither scheduled (based on the set respiratory frequency) nor initiated by the patient" giving the impression of tachypnea or hyper ventilation [6].

It is possible to identify auto-triggering by looking at the pressure/time waveform. Auto-triggering shows in the pressure/ time waveform, a lack of airway pressure drop or variations in the flow/time waveform at the begining of the inspiratory phase [14][Figure 2]. Complications associated with this type of PVA include dynamic hyper inflation and respiratory alkalosis, which may lead to confusion in the clinical team. Due to this, when auto-triggering is suspected, it is important to evaluate if there is inspiratory effort in the pressure/time waveform, the breathing pattern, the general condition, sedation levels, cuff pressure 
levels, endotracheal tube position, presence of condensation in the circuit, the programming of trigger sensitivity, cardiac oscillations and the type of ventilator used [6,19-21].

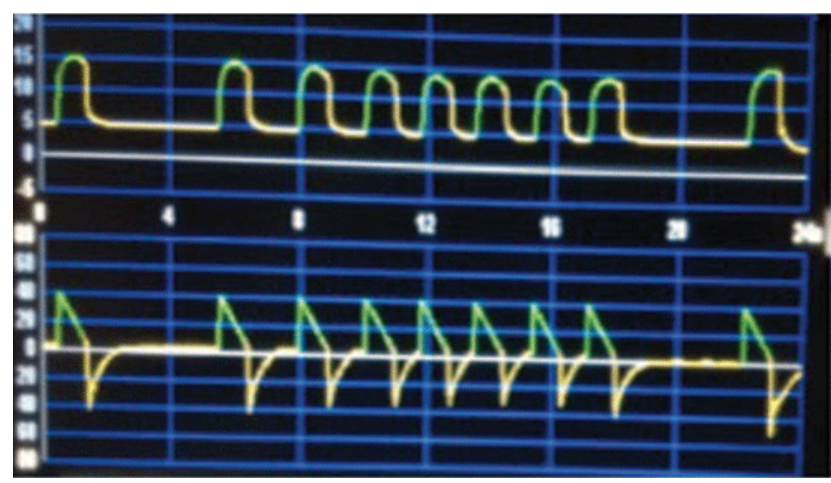

Figure 2: Auto-triggering caused by leak in the circuit. Note that there is no drop of the airway pressure in the pressure/time waveform (Upper waveform) at the beginning of the inspiratory phase which means that the breaths are not patient triggered

In a prospective randomized study Carteauxet al evaluated the incidence of auto-triggering generated by invasive mechanical ventilators, that included an algorithm to be used as non-invasive ventilator, versus non-invasive ventilators [21]. In a sample of 15 patients, they performed 3 consecutive measurements of 20 minutes each, where they ventilated patients first using an invasive ventilator, with the option to be used as non-invasive deactivated; second, with the option activated and then with a non-invasive ventilator technique, using an oronasal interface in all the cases. When a non-invasive ventilator was used, the incidence of auto-triggering was significantly lower in the invasive ventilator compared with the non-invasive option activated, but this also showed a decrease in the incidence of auto-triggering. Another interesting result of the study was that $27 \%$ of the 15 patients evaluated, presented an $\mathrm{AI}>10 \%$ when invasive ventilation with the option of the algorithm disabled was used, $13 \%$ with the option activated and $0 \%$ when a noninvasive ventilator was used. It is important to remember that one of the main characteristics of non-invasive ventilation is that they compensate leaks, so the appearance of auto-triggering will not be a problem when this type of ventilator is used. Therefore, in the case of invasive ventilation, the risk of auto-triggering will always be present, even though the algorithm to be used as noninvasive is included.

As it was mentioned, another cause of auto-triggering are cardiac oscillations. Imanaka et al evaluated the effect of flow triggering versus pressure triggering in 104 patients after cardiac surgery, finding that when flow triggering was used, the incidence of auto-triggering resulting from cardiac oscillations was higher, which is associated with high ventricular filling pressures, high cardiac output, and increased heart size [19]. A proper programming does not cause a great effort for the patient, but neither auto-triggering.

\section{Double Triggering}

Double triggering is a type of asynchrony that can occur in modes controlled by pressure and volume [4]. Thille et al found that factors associated with a high incidence of double-triggering were: a low pao2/FIO2 ratio, ACV (assist-control ventilation mode), a shorter inspiratory time, a high maximal inspiratory pressure, and a high level of PEEP $[4,22]$. Double trigger can be defined as "two cycles separated by a very short expiratory time, defined as less than one-half of the mean inspiratory time, the first cycle being patient-triggered" $[4,15,24]$. This type of PVA can occur when neural inspiratory time is longer than the inspiratory time set on the ventilator2. This may trigger a second breath by the ventilator if the patient inspiratory effort continues after the ventilator cycle to expiratory phase and if these efforts generated by the patient are able to overcome the trigger threshold programmed by the clinician [4]. Other studies agree with the findings of Thilleet al., Takioka $\mathrm{H}$ et al also demonstrated that there is a relationship between short-term inspiratory times programmed in the ventilator and double triggering, which is also related to a high ventilatory demand $[4,23]$. Double triggering can cause great harm to the ventilated patient because if the patient doesn't have enough expiratory time, the tidal volume delivered from the second breath will add to the tidal volume from the first breath which may double the tidal volume delivered to the patient causing over distension, volutrauma and barotrauma. [Figure 3]

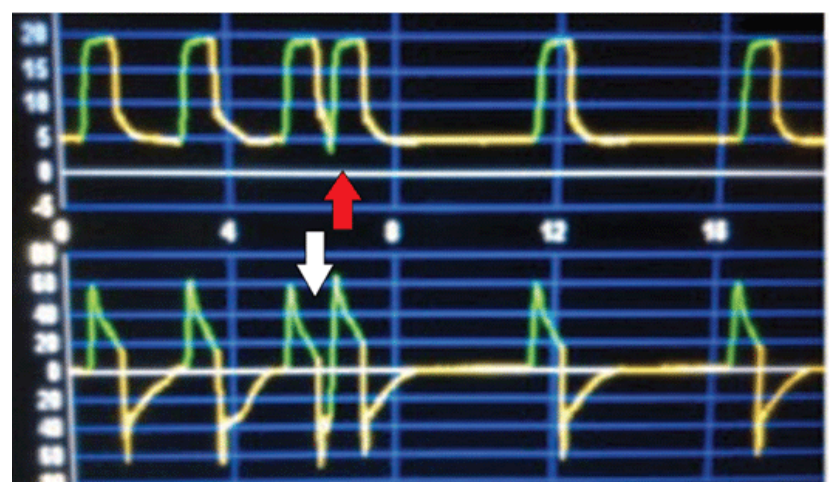

Figure 3: Red arrow show double-triggering in the pressure/time waveform. White arrow show double-triggering in the flow/time waveform

It is important to realize that the first cycle delivered can be triggered by patient effort or the ventilator. Liao et al classified the double triggering in DT-P (double triggering where the patient triggers these first cycle), DT-A (double trigger where the first cycle is triggered byauto-triggering) and DT-V (when the first cycle is triggered by the ventilator according to the programmed criteria) [15]. The results in the same study showed that the DT-P is associated with short inspiratory time and that a correct programing of the inspiratory time decreases or even eliminate the presence of double triggering. On the other hand, the DT-V is related to the programming of respiratory rate, and could be solved by decreasing the inspiratory time or changing to a spontaneous mode. These techniques were successfully applied in 14 patients. 


\section{Reverse Triggering}

Reverse triggering is a poorly recognized type of PVA. Muriaset al defined Reverse trigger as "a type of PVA in which the patient's respiratory center is activated in response to a passive insufflations of the lungs" [25]. During reverse triggering, there is a delay between the start of the machine-triggered breath and the start of the patient's inspiratory effort. As a result, the patient's effort usually persists when the inspiratory phase is completed, which could generate a double triggering if the inspiratory effort of the patient is able to overcome the threshold of programmed sensitivity [25].

The pressure/time waveform shows a breath that is initiated by the ventilator (there is no airway pressure drop at the beginning of the breath), and/or also an airway pressure drop during the inspiratory phase and part of the expiratory phase produced by the activation of the respiratory of the patient with the consequent contraction of the inspiratory muscles. In addition, an amputation or deformation of the peak expiratory flow evidenced in the flow / time waveform product of the inspiratory effort of the patient is observed [25][Figure 4]. Akoumianakiet al indicated that this type of PVA can induce a continuous plyometric contraction of the diaphragm [26]. This contraction is associated with cytokine release and damage of muscle fibers. In addition, it can produce and increase of the respiratory work, oxygen consumption, confusion in the monitoring of plateau pressure and increased in the plateau pressure levels in modes controlled by volume.

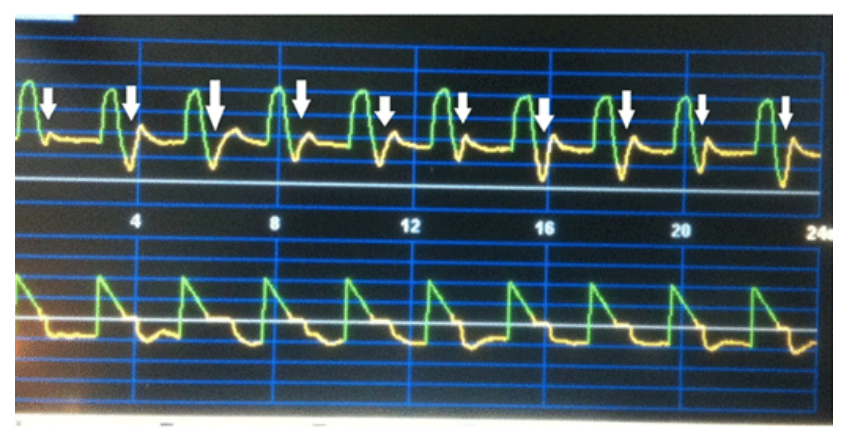

Figure 4: White arrows show reverse triggering in the pressure/time waveform

\section{Flow Asynchronies}

This type of PVA occurs when the programmed flow in the ventilator does not meet the patient's flow demands, causing an increase in the work of breathing. It occurs frequently in that ventilated with volume-controlled modes, with acute respiratory failure and high ventilatory demand. Flow asynchrony is less frequent in patients ventilated in pressure-controlled modes because in the flow is variable [27-29]. Kalletet al compared the effect of pressure-controlled ventilation and volumecontrolled ventilation on the work of breathing of patients with acute lung injury and respiratory distress syndrome [27]. They found that respiratory work was significantly lower in pressure control modes. Yang et al compared respiratory effort produced in volume-assist/control ventilation versus pressure-assist/ control ventilation in patients with acute respiratory failure by measuring P0.1 (pressure generated in the first $100 \mathrm{~m} . \mathrm{sec}$ ) [28]. The results obtained showed that P0.1 decreased in a $25 \%$ when pressure-assist/control ventilation was used, in addition to decreasing patient discomfort. Macintyre et al induced a flow asynchrony, by decreasing the programmed flow $50 \%$ in 16 stable patients ventilated with volume-assist/control ventilation and subsequently attempted to correct it in two ways [30]. (1) Reestablishing the flow that was initially programmed by adding $25 \%$ more of the original value and (2) using a pressurelimited mode. Measurements of respiratory effort were made by calculating the pressure-time product and the breathing pattern. Both strategies demonstrated a significant decrease in flow asynchrony: however, the strategy that involved switching to a pressure controlled mode was more effective in those patients who presented a severe degree of asynchrony. It is worth noting that in most studies, work of breathing and discomfort caused by inadequate flow decreases with pressure-controlled modes in patients with a high ventilatory demand, because they modify their effort and need for flow in each respiratory breath [29]. For this reason, programming a fixed flow level (such as occurs in volume-controlled modes) would produce PVA not providing and adequate flow.

As Branson mentioned "the scalloped-out portion of the pressure/time waveform during a patient-triggered volume breath, is a well-recognized sign of flow asynchrony. Nilsestuen and Hargett also mentioned how to identify flow asynchrony [31]. They explained that "as patient effort increases, the peak flow set on the ventilator no longer meets the patient's flow demand and the airway pressure waveform becomes progressively dished out. This phenomenon is informally recognized as "flow starved" [8] [Figure 5].

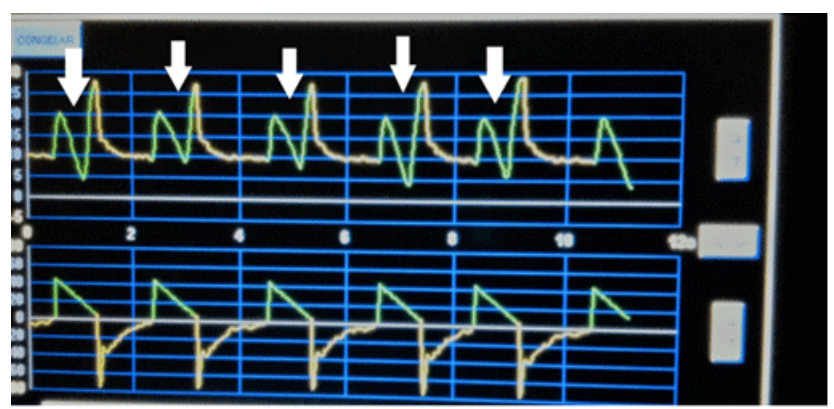

Figure 5: White arrows show flow asynchrony in the pressure/time waveform

\section{Premature Cycling}

The cycle variable can be defined as "the variable (usually pressure, volume, flow, or time) that is measured and used to end inspiration (and begins expiratory flow) [10]. Premature cycling as well as double triggering is a type of asynchrony that occurs when the patient's neural inspiratory time is greater than the inspiratory time programmed in the ventilator [8]. The difference, with the double triggering, is that in premature cycling the inspiratory effort of the patient is not enough to trigger a second breath. Premature cycling produces a significant decrease in 
airway pressure, which can be seen immediately after the end of the inspiratory phase programmed in the ventilator, accompanied by an increase of the inspiratory flow which can be seen in the flow/time waveform [32][Figure 6]. This type of PVA could be confused with an ineffective effort during the expiratory phase, with the difference that premature cycling responds to changes in programmed inspiratory time or cycling; where as ineffective efforts responds to changes in the level of PEEP, sensitivity or assistance levels [23]. Another difference is that in premature cycling the drop of the airway pressure occurs immediately after the inspiratory phase has ended, indicating that the patient's inspiratory effort continues. Takioka et al evaluated the effect of cycling (expiratory sensitivity) at $45 \%, 35 \%, 20 \%, 5 \%$ and $1 \%$ of the peak inspiratory flow in 8 ventilated patients under supportive pressure mode [23]. They found that there was an increased respiratory work and asynchrony (premature cycling and double triggering) when the ventilator cycled at 45 and $35 \%$ of maximal inspiratory flow $(0.31 \pm 0.12 \mathrm{~J} / \mathrm{L}$ versus $0.51 \pm 0.11 \mathrm{~J} /$ $\mathrm{L}$ with cycling criterion of $1 \%$ and $45 \%$, respectively). The greater percentage of cycling criteria, the shorter the inspiratory time [8].Therefore, when patient is ventilated with pressure support ventilation, is very important consider this aspect in the setting to ensure a proper ventilation.

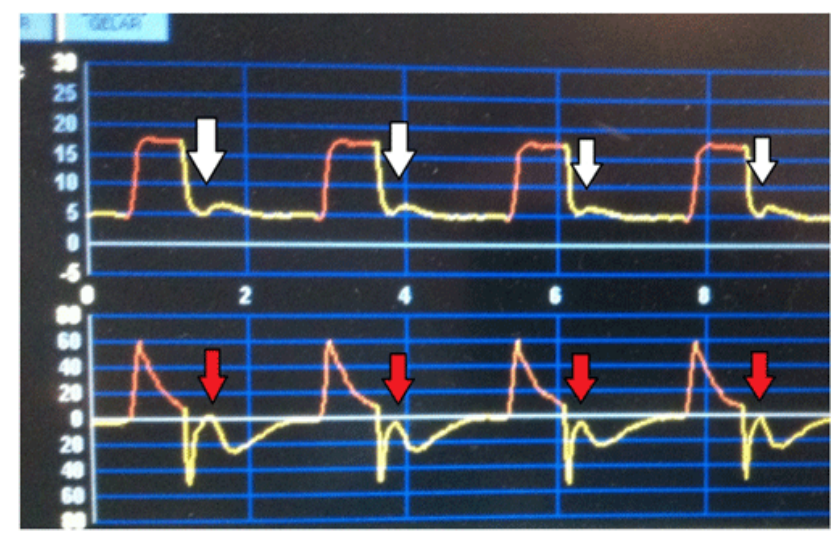

Figure 6: Example of premature cycling. White arrows show an inspiratory effort that continues after the inspiratory phase ended in the pressure/time waveform. Red arrows show a sudden change in the expiratory flow caused by the inspiratory effort of the patient

\section{Delayed Cycling}

TDelayed cycling occurs when the inspiratory time programmed in the mechanical ventilator exceeds the patient neural inspiratory time [6]. This means that the system continues in the inspiratory phase, once the patient's inspiratory effort has ended, which decreases the time available for the expiratory phase. This may produce an activation of the patient's expiratory muscles before the established cycling criteria (active exhalation), air trapping, dynamic hyperinflation and PEEPi, which may increase the work of breathing and cause ineffective efforts [3234]. Parthasarathy et al mentioned that "a delay in relaxation of the expiratory muscles could cause them to remain active during the early phase of the next inspiration, and by opposing the downward motion of the diaphragm could hinder the efficacy of the subsequent inspiratory effort."
This type of asynchrony is common in COPD patients because of PEEPi and a short expiratory time. In these cases, an effective solution would be decreasing the inspiratory time in controlled modes such as pressure assist/control ventilation and Synchronized Intermittent Mandatory Ventilation (SIMV) $[33,34]$. The inspiratory time can be decrease, also, by modifying the cycling criteria in pressure support ventilation [23]. So, by modifying the cycling criteria ineffective efforts and active expiration caused by delayed cycling can be prevent [35,36].

Delayed cycling is evidenced in the pressure/time waveform as an increase in airway pressure near the end of the inspiratory phase and in the flow/time waveform by a sudden decrease in inspiratory flow, resulting from activation of the expiratory muscles [Figure 7].

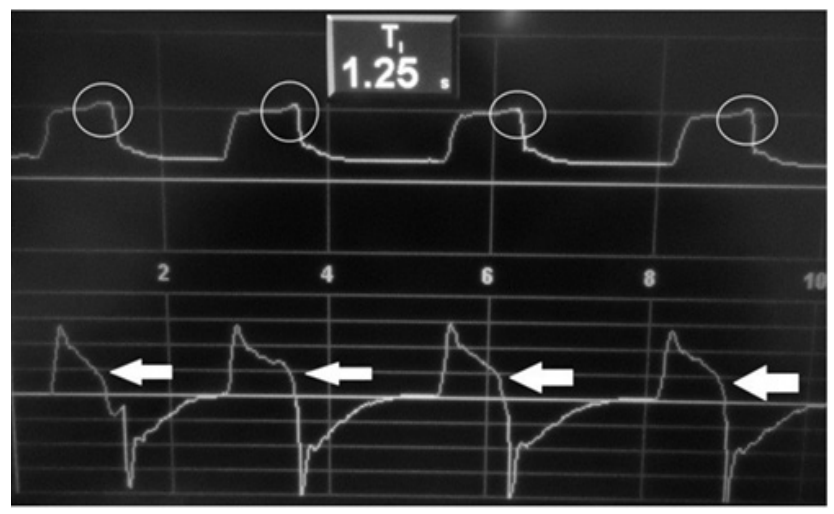

Figure 7: Example of Delayed cycling. White circles show, in the pressure/time waveform, an increase in airway pressure near the end of the inspiratory phase caused by the contraction expiratory muscles. White arrows show, in the flow/time waveform, a rapid decrease in inspiratory flow resulting from activation of the expiratory

\section{Conclusion}

Identifying patient-ventilator using waveform analysisis a very useful and important skill that every health care professional that work in the ICU should develop in order to prevent complications that may affect the outcome of the mechanically ventilated patient. It is also important to establish standard definitions for all types of PVAs, considering that different definitions are used to describe the same problem.

\section{References}

1. Arellano D. Ventilación mecánica: generalidades y modalidades tradicionales. Kinesiología. 2006;25(4):17-24.

2. Epstein SK. How often does patient-ventilator asynchrony occur and what are the consequences? Respir Care. 2011;56(1):25-38. doi: 10.4187/respcare.01009

3. Georgopoulos D, Prinianakis G, Kondili E. Bedside waveforms interpretation as a tool to identify patient ventilator asynchronies. Intensive Care Med. 2006;32(1):34-47.

4. Thille AW, Rodriguez P, Cabello B, Lellouche F, Brochard L. Patientventilator asynchrony during assisted mechanical ventilation. Intensive Care Med. 2006;32(10):1515-1522. 
5. Kacmarek RM. Mechanical ventilation competencies of the respiratory therapist in 2015 and Beyond. Respir Care. 2013;58(6):1087-1096.

6. Branson RD, Blakeman TC, Robinson BR. Asynchrony and dyspnea. Respir Care. 2013;58(6):973-989.

doi: $10.4187 /$ respcare.02507

7. MacIntyre NR. Patient-Ventilator Interactions: Optimizing Conventional Ventilation Modes. Respir Care. 2011;56(1):73-84. doi: 10.4187/respcare.00953

8. Gilstrap D, MacIntyre NR. Patient-Ventilator Interactions: Implications for clinical Management. Am J Respir Crit Care Med. 2013;188(9):10581068. doi: $10.1164 / \mathrm{rccm} .201212-2214 \mathrm{CI}$

9. Dhand R. Ventilator Graphics and Respiratory Mechanics in the Patient with Obstructive Lung Disease. Respir Care. 2005;50(2):246-261.

10. Ramirez II, Arellano DH, Adasme RS, Landeros JM, Salinas FA, Vargas AG, et al. Ability of ICU health-care professionals to identify patient-ventilator asynchrony using waveform analysis. Respir Care. 2017;62(2):144-149.

11. Hess DR. Patient-Ventilator Interaction During Noninvasive Ventilation. Respir Care. 2011;56(2):153-165.

12. Chao DC, Scheinhorn DJ, Stearn-Hassenpflug M. Patient-ventilator trigger asynchrony in prolonged mechanical ventilation. Chest. 1997;112(6):1592-1599.

13. de Wit M, Miller KB, Green DA, Ostman HE, Gennings C, Epstein SK Ineffective triggering predicts increased duration of mechanical ventilation. Crit Care Med. 2009;37(10):2740-2745.

14. Thille AW, Brochard L. Promoting Patient-Ventilator Synchrony. Clin Pulm Med. 2007;14(6):350-359.

15. Liao KM, Ou CY, Chen CW. Classifying Different Types of Double Triggering Based on Airway Pressure and Flow Deflection in Mechanically Ventilated Patients. Respir Care. 2011;56(4):460-466. doi: $10.4187 /$ respcare. 00731

16. de Wit M, Pedram S, Best AM, Epstein SK. Observational study of patient-ventilator asynchrony and relationship to sedation level. J Crit Care. 2009;24(1):74-80. doi: 10.1016/j.jcrc.2008.08.011

17. Leung P, Jubran A, Tobin MJ. Comparison of assisted ventilator modes on triggering, patient effort, and dyspnea. Am J Respir Crit Care Med.1997;155(6):1940-1948.

18. Bernstein G, Knodel E, Heldt GP. Airway leak size in neonates and autocycling of three flow-triggered ventilators. Crit Care Med. 1995;23(10):1739-1744.

19. Imanaka H, Nishimura M, Takeuchi M, et al. Autotriggering caused by cardiogenic oscillation during flow-triggered mechanical ventilation. Crit Care Med. 2000;28(2):402-407.

20. Ghamloush M, O'Connor H, White A. Patient-Ventilator interaction in the Long-Term Acute-Care Hospital. Respir Care. 2011;56(2):207-213.

21. Carteaux G, Lyazidi A, Cordoba-Izquierdo A, et al. Patient-ventilator asynchrony during noninvasive ventilation. Chest. 2012;142(2):367376.
22. Sassoon CSh. Triggering of the Ventilator in Patient-Ventilator Interactions. Respir Care 2011;56(1):39-51. doi: 10.4187/ respcare.01006

23. Takioka H,Tanaka T, Ishizu T, Fukushima T, Iwaki T, Nakamura Y, et al. The effect of breath termination criterion on breathing patterns and the work of breathing during pressure support ventilation. Anesth Analg. 2001;92(1):161-165.

24. Carlucci A, Pisani L, Ceriana P, Malovini A, Nava S. Patient-ventilator asynchronies: may the respiratory mechanics play a role? Crit Care. 2013;17(2):R54. doi: 10.1186/cc12580

25. Murias G, de Haro C, Blanch L. Does this ventilated patient have asynchronies? Recognizing reverse triggering and entrainment at the bedside. Intensive Care Med. 2016;42(6):1058-1061. doi: 10.1007/ s00134-015-4177-3

26. Akoumianaki E, Lyazidi A, Rey N, Matamis D, Perez-Martinez N, Giraud R, et al. Mechanical ventilation induced reverse-triggered breaths: a frequently unrecognized form of neuromechanical coupling. Chest. 2013;143(4):927-938.

27. Kallet RH, Campbell AR, Alonso JA, Morabito DJ, Mackersie RC. The effects of pressure control versus volume control assisted ventilation on patient work of breathing in acute lung injury and acute respiratory distress syndrome. Respir Care. 2000;45(9):1085-1096.

28. Yang LY, Huang YC, Macintyre NR. Patient-ventilator synchrony during pressure-targeted versus flow-targeted small tidal volume assisted ventilation. J Crit Care. 2007;22(3):252-257.

29. Cinnella G, Conti G, Lofaso F, Lorino H, Harf A, Lemaire F. Effects of assisted ventilation on the work of breathing: volume-controlled versus pressure-controlled ventilation. Am J Respir Crit Care Med. 1996;153(3):1025-1033.

30. MacIntyre NR, McConnell R, Cheng KC, Sane A. Patient-ventilator flow dyssynchrony: flow-limited versus pressure-limited breaths. Crit Care Med. 1997;25(10):1671-1677.

31. Nilsestuen JO, Hargett KD. Using ventilator graphics to identify patientventilator asynchrony. Respir Care. 2005;50(2):202-234.

32. de Wit M. Monitoring of Patient - Ventilator interaction at the bedside. Respir Care. 2011;56(1):61-72. doi: 10.4187/respcare.01077

33. Parthasarathy S, Jubran A, Tobin M. Cycling of inspiratory and expiratory muscle groups with the ventilator in airflow limitation. Am J Respir Crit Care Med. 1998;158(5):1471-1478.

34. Gentile MA. Cycling of the Mechanical Ventilator Breath. Respir Care. 2011;56(1):52-60.

35. Hess DR. Ventilator waveforms and the physiology of pressure support ventilation. Respir Care. 2005;50(2):166-186.

36. MacIntyre NR, Ho LI. Effects of initial flow rate and breath termination criteria on pressure support ventilation. Chest. 1991;99(1):134-138. 